\title{
A Study of Rapid Business Application Development in the Cloud
}

\author{
Jie Cui ${ }^{1}$, Jing Min $\mathrm{Xu}^{1}$, Huiping $\mathrm{Lin}^{2}$, Weiping $\mathrm{Li}^{2}$, and $\mathrm{Zi} \mathrm{Mu} \mathrm{Sun}{ }^{2}$ \\ ${ }^{1}$ IBM Research - China, Beijing, China \\ ${ }^{2}$ School of Software and Microelectronics, Peking University, Beijing, P.R.China \\ cuijie@cn.ibm.com, xujingm@en.ibm.com, \\ linhp@ss.pku.edu.cn, wpliass.pku.edu.cn, zmsun@ss.pku.edu.cn
}

\begin{abstract}
The emergence of Cloud Computing promotes a rapid business application development (RBAD in short) paradigm, which enables non technical developers to create applications using the interactive and feature-rich tools via browser without caring about the underlying infrastructure. In this paper, we review the RBAD platforms in marketplace with various dimensions. To better understand their feasibility compared to the traditional software development, we conducted a user study with 12 participants creating application on 3 selected RBAD platforms. Analyzing their behaviors and feedback from different development perspectives, we design a conceptual business application model for RBAD platform, identify the best practice and gaps, and propose 6 design recommendations for RBAD system.
\end{abstract}

Keywords: Cloud, Business Application Development, User Study, PaaS.

\section{Introduction}

Cloud Computing dramatically reduces the complexity of owning infrastructure, software and services through on demand resources provisioning over virtualization and pay-as-you-go usage pattern $[1,2]$. It also promotes a new paradigm in which business application is developed online and can be conducted by so called business developers who know the business knowledge best but with limited programming skills. Some serious business developers may possibly write simple script for enhanced business logic, just like they did in editing formula upon spreadsheet or defining email-filters. This new development approach greatly takes advantages of Cloud Computing and Software as a Service (SaaS) model in which vendors host and provide access to a software application over Internet [18]. Different from the traditional enterprises application development and deployment with a dedicated IT architecture and costly resource allocation, developers can build business applications faster and easier than ever without installing any tools, caring about the environment maintenance and compromising scalability, performance and security.

As the IT infrastructure gets increasing complex and new development techniques are emerging, this paradigm is becoming more and more attractive to small to middle size company as well as departments within larger organizations, because it lowers 
the barrier of creating applications and can greatly reduce the development complexity and cost, without new investment on capitals and human resources.

There are many such rapid business application development (RBAD in short) platforms in market ranging from the ones which can step you through a drag-anddrop interface to build form-based application such as Wufoo [15], JotForm [18], FormAssembly [12], Zoho Creator [14], Quickbase [19] etc, to sophisticated platforms like Force.com [6] (a product of Salesforce.com), WaveMaker [16] and Bungee Connect [20], which can integrate a company's existing programs through Web interfaces and provide libraries, scripting or server side programming language for business process customization. As more big companies such as Google, Microsoft, IBM and Amazon join this fray, fostering the rapid market growth, we envision this new paradigm as a potential shift for business application development and corporate IT and might be disruptive to the traditional software development.

To investigate the feasibility of this programming and business model and also analyze requirements and feedback from real user experience, we overview the emerging marketplace with a variety of dimensions. In particular, a user study is designed and conducted on the selected representative platforms: Zoho Creator, Force.com and WaveMaker. Furthermore, we explore and discuss the following research question: What are the associated implications and design recommendations for RBAD system?

The structure of the paper is as follows. Section 2 presents the related work. A review of the RBAD platforms in market is given in Section 3. Section 4 details our user study design and conduction, and is followed by the result analysis in Section 5 . Section 6 further discusses the research questions with critical insights gained. Finally, we summarize the paper and outlook the future work in Section 7.

\section{Related Work}

Gautam Sharoff named this new paradigm as Dev 2.0 [3], where the line between users and developers is blurred, and an application is available to all stakehoders through the lifecycle as it evolves. More generally, it is referred as Platform as a Service (PaaS) model which takes SaaS model one step further, providing end to end application development environment from editing code to debugging, deployment, runtime and management $[4,5]$. PaaS model is built upon Cloud infrastructure and uses a multi-tenant deployment and development tools providing important value for developers to focus on development and innovation, while eliminating the burden of configuring servers, implementing management tools, wresting with the storage, OS and middlewares, and dealing with the network interfaces. Force.com is an industry leading PaaS offering. It further categorizes PaaS solutions into 4 categories [23]: 1) Social application platforms (e.g., Facebook [24]) which provide APIs so third parties can write new application functionality that is made available to all users. 2) Raw compute platforms like Amazon Web Services [21] which provide storage, processor, and bandwidth as a service. 3) Web application platforms like Google App Engine [22] which provide APIs and functionality for developers to build Web applications. 4) Business application platforms like Force.com which provide application infrastructure specifically geared toward transactional business applications. 
In this paper, we are more interested in the last PaaS type used by business developer instead of professional developers who are mostly targeted by the other three types. Although there are quite a lot researches in investigating the issues of developing software in a Cloud environment or the design factors of PaaS platforms $[25,26,27]$, to our best knowledge, there has been relatively little research giving systematic survey and exploratory study on RBAD in the Cloud to discuss the feasibility and real user feedback. Therefore, our paper can complement to this research field.

\section{Overview of the RBAD Platform}

We totally studied 9 RBAD platforms and reviewed them from a variety of aspects as follows: 1) target user, 2) target application, 3) functions, 4) developing lifecycle, 5) differences from local IDE (Integrated Development Environment) .

According to our survey, the RBAD platforms can be divided into two categories, i.e., 1) online form builder with or without database (DB) support, and 2) sophisticated platforms supporting customized applications with more complicated business processes.

\subsection{Online Form Builder}

Two types of online form builders were identified depending on whether they have the support of DB operation. Totally 7 online form builders were selected and studied.

\section{Online Form Builder without DB Support}

Online form builder without DB support is more or less an online service that helps users create forms without writing codes and provides an interface for collecting data, but little DB operations are allowed, e.g., Wufoo [15], JotForm [18], FormAssembly [12], and Iceberg[28]. Their target users are those that have little programming skills and little time to learn.

2. Online Form Builder with DB support

Online form builders with DB support allow users to build online database application with CRUD (create, retrieve, update and delete) controllers that can deal with more complicated data. Three platforms, namely QuickBase [19], DabbleDB [13], and Zoho Creator [14] were studied. Comparing with online form builders without DB support, their target applications usually include more complicated data and report analysis. They provide more functions to users such as creating the database, sharing the information and carrying out report analysis.

\subsection{Sophisticated RBAD Platform}

Two more powerful platforms Force.com [6] and WaveMaker [16] were studied as well to see how it can support customized business logic and presentation.

Force.com platform is a PaaS product owned by Salesforce.com, who delivers CRM solutions to customers over internet as service. The platform allows external developers to create add-on applications that integrate into the main Salesforce 
application and are hosted on Salesforce.com's infrastructure. Force.com targets at both business developer and technical developers.

WaveMaker is available as a traditional product that is installed on a developer's computer (desktop) or as a software as a service that is accessible via the web (cloud). The difference with other platforms is that it can import local database and automatically generate data model you want to access on WaveMaker.

\section{User Study}

We designed a typical online airline reservation application scenario and conducted a user study for developing it on the representative RBAD platforms to identify advantages and functional gaps comparing to traditional application development from real user feedback.

\subsection{Online Airline Reservation Application}

The application scenario is quite straight forward and can be simply described as the following steps.

1. User is allowed to search for the flight and airfare by selecting origin, destination, dates and flight type.

2. User can select an available one and create an order.

3. After assigning the passengers information and inputting the payment information, user can submit the order.

4. The credit card will be validated and then a notification email will be automatically generated and sent to the user.

We choose this application for testing the platform functions not only because it represents the typical features in reservation systems including search, list and order, but also because it covers most of the test points we designed for RBAD platforms as follows.

1. The diversity of the field types in business objects. E.g., the flight information contains field type of date, text, currency and integer etc.

2. The correlation of business objects. E.g., an order instance associating flight information and probably user information would be created after user submits the order.

3. Navigation logic in the application. This application includes a typical sequential page flow from the starting search page to the final order page, in which parameters are passed to ensure the page linkage and integrity. In traditional Web application development such as using JSF (Java Server Face), page flow can be explicitly configured in an XML file which indicates the "from" and "to" pages and associated navigation conditions. We will see how it can be achieved in RBAD platforms.

4. The ability to integrate external application. We intentionally provided two external Web Services based on SOAP and REST respectively, one to verify user credit card, and the other to send email notification. 


\subsection{Participants and Procedures}

We recruited 12 volunteers (10 male, 2 female) of college students from different majors (e.g., business, mathematics, and computer science (CS)). The reason that we also hired the students with programming background is to testify the advanced functions that the platform provides such as programming for customized logic. 4 students whose major is CS experienced with software development project for 2 to 4 years. Other students didn't have much Web programming experience.

We balanced the programming experience across the three groups, see table 1 .

Table 1. User study group assignment

\begin{tabular}{|c|c|c|c|}
\hline & Zoho Creator Group & Force.com Group & WaveMaker Group \\
\hline Participants & 4 & 4 & 4 \\
\hline CS students & 1 & 1 & 2 \\
\hline
\end{tabular}

For each group, there was a facilitator assigned to manage the whole procedure and observe their behaviors. Firstly an overview of the tasks they would be carrying out was given, followed by a 30 minutes tutorial, a more concise version of the quick start guide of each platform, with a hands-on session to get them familiar with the functions of the platform. Then a questionnaire adapted from [7] to the specific task of RBAD was completed by participants to identify their expectations of the outcomes and emotional reactions to the unfamiliar software.

A requirement documents describing the application scenario (Section 4.1) was provided to the participants. Also, an additional tutorial would be given when participates made no progress. After participants confirmed they completed the task, a focus group discussion was conducted to gather their feedback, and a survey questionnaire designed to identify the issues from different development perspectives was finally completed.

\subsection{Environment}

Zoho creator and Force.com platform provide free version and 30 days free trial version respectively with almost all the functions claimed except advanced performance and security insurance. Also, free registered user can directly access WaveMaker cloud version. Therefore, a shared account was applied on above platforms by each group. Additionally, each group was separately located and was allowed to discuss the application design, including business objects construct, business logic, UI design and page flow, which was guided by the CS background students. All the participants were required to develop as least one component of the application and the procedure was recorded. The connection speed to internet was around $1 \mathrm{M}$ but sometimes it was deceased due to network traffic. 


\section{Result Analysis}

We observed how participants approached the task and classified their development activities into 4 types: 1) data (setting up data model on Zoho Creator and Force.com or connecting local DB on WaveMaker, viewing or updating $\mathrm{db}$ records and etc) 2) business logic (defining or testing business objects association, workflow and app integration) 3) UI (pages and page flow creation, editing, viewing or testing) 4) problem solving (referring back to the tutorial or online help, asking for partner's or facilitator's help, and debugging a error encountered). Since the facilitators with strong CS background had a pre in-depth study of the platform, they can be quite easy and accurate to detect the distinctions of the above behaviors.

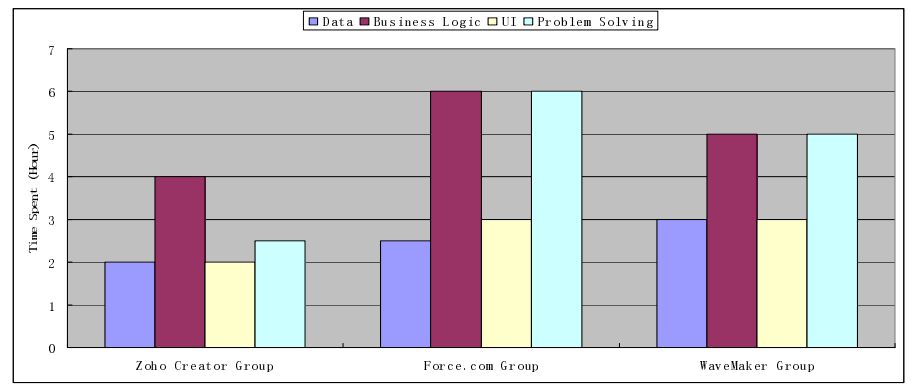

Fig. 1. Development time spent in different activities

Figure 1 shows the time spent in those four types of behaviors of each group. Basically, user spent most of time in developing business logic and UIs, that's also very common in traditional development paradigm and specially in this experiment which evolved scripting (e.g., Deluge on Zoho Creator) or additional programming (e.g., Apex [8] and Visualforce [9] on Force.com, JavaScript and Java on WaveMaker). Creating data structures seem not time consuming, suggesting the facilitation for building forms is very effective, e.g., drag-and-drop tool (Zoho Creator), point-and-click tool (Force.com) and local DB connection (WaveMaker). Additionally, we noticed users on Force.com spent significantly more time on problem solving than the other two so that we believe there is a relative steep learning curve for novice users on Force.com. That is also reflected in a large amount of documentation on developerforce [10] that details the advanced functionalities.

\subsection{Data Development}

Data model is the basic construct of an application especially for the business application which mainly manipulates information entities that transform in the business to achieve the organization goal. Since the majority of the audience are business developers or non-technical administrators, many RBAD platforms use terminology that is familiar to them, e.g, it is avoided to use "DB Tables" and "Rows" instead of "Objects" and "Records" within the context of Force.com; Application 
creation is started from "Form", "Fields" and "View" which are more visualized from the end user view on Zoho Creator. Compared to the traditional DB development, Table 2 summarizes the function supportability of the 3 platforms.

Table 2. Data development supportability

\begin{tabular}{|l|l|l|l|}
\hline & Zoho Creator & Force.com & WaveMaker \\
\hline Related objects & supported & supported & supported \\
\hline Graphic widgets & supported & supported & supported \\
\hline Data validation & supported & supported & supported \\
\hline CRUD operation & supported & supported & supported \\
\hline Data import & XLS,CSV, MDB & CSV & Local DB \\
\hline
\end{tabular}

\subsection{Business Logic Development}

The powerfulness of the supporting business logic layer is a good demonstration point in terms of platform flexibility. The business logic orchestrates interaction among business entities, therefore controls the navigation of user interface. The server side scripting and programming capabilities allow advanced user to develop relative complex and customized business logic on the 3 platforms. We further categorized the business logic support in Table 3.

Table 3. Business logic development supportability

\begin{tabular}{|l|c|c|c|}
\hline & Zoho Creator & Force.com & WaveMaker \\
\hline Form actions & supported & supported & supported \\
\hline Workflow editor & not supported & supported & not supported \\
\hline App integration & $\begin{array}{c}\text { partially } \\
\text { supported }\end{array}$ & supported & supported \\
\hline Programming & Delug & $\begin{array}{c}\text { Apex, } \\
\text { VisualForce }\end{array}$ & $\begin{array}{l}\text { Javascript } \\
\text { Java Service }\end{array}$ \\
\hline
\end{tabular}

Regarding to application integration, Zoho Creator provides REST as well RPC style Data APIs that allows external websites to collaborate with its applications, but with limited support for calling external REST or Web Service. Force.com has the two-way integration approach which means the external application can access Force.com data and logic through Web Service API, and more facilitation such as data validation, workflow, Apex triggers and the Force.com security scheme can help user to integrate external application within Force.com application. While WaveMaker provides the user interface for importing REST, Web and RSS services to the application which is more convenient than writing code. 


\subsection{UI Development}

RBAD platforms provide metadata-driven, automatically generated user interfaces and page layouts which impress the participants most. They found it was fun to drag and drop visual widgets and compose or layout them in the canvas. More advanced functions are summarized in Table 4.

Table 4. UI development supportability

\begin{tabular}{|c|l|c|c|}
\hline & Zoho Creator & Force.com & WaveMaker \\
\hline Drag \& drop & supported & supported & supported \\
\hline Custom theme and layout & supported & supported & supported \\
\hline JavaScript & not supported & supported & supported \\
\hline CSS & not supported & supported & supported \\
\hline Page flow configuration & not supported & $\begin{array}{c}\text { not } \\
\text { Supported }\end{array}$ & not Supported \\
\hline
\end{tabular}

Visualforce [9], a component-based framework in MVC paradigm provided by Force.com, helps users to create and deploy fully custom user interface. Not like fully customized UI on Force.com, the other two platforms provide most necessary UI customization capabilities such as theme or layout selection, or user defined CSS (WaveMaker) etc.

\subsection{Summary}

Analyzing the comments from the participants, we summarize the 3 platforms as below.

1. Zoho Creator is the most intuitive platform on which participants saved half of the time than the other two. Programming script was also an enjoyable task for participants and could be quickly referenced in the documentation, but not being able to call external Web Service is an imperfection from application integration view.

2. Force.com is the most powerful and comprehensive platform among them. All the functional requirements of the airline reservation can be implemented on Force.com including form creation, Web Service calling and page flow. But it took participants much longer time to build the application and they complained about the long learning process and always got frustrated in facing errors without quickly identifying the problems and always lost in the numerous documents Force.com provides.

3. WaveMaker targets more technical people who should have the knowledge of database and programming. The performance was not being satisfied since every action was needed to manually saved for a long time and data was always lost which heavily decreased the development productivity. 


\section{Conclusion and Future Work}

In this paper, we have presented an overview of the new and promising development paradigm namely RBAD and a user study for the representative RBAD platforms. Through the analysis of user behavior and feedback, we contributed to the evidence and identification of both advantage and gaps over traditional development. We also distilled 6 design recommendations for future RBAD and PaaS platform. We plan to further develop the work in three directions. Firstly, we will carry out additional empirical work to explore other functions such as security, performance, testing and deployment with larger set of RBAD platforms. Secondly, we will begin to develop lightweight tools to validate the business application model proposed and apply the design recommendations. Finally, we will expand the study to the PaaS domain and larger development community to identify problems and solutions

Acknowledgments. We sincerely thank the participants for volunteering their time to our user study.

\section{References}

1. Armbrust, M., Fox, R.G.A., et al.: Above the clouds: A berkeley view of cloud computing. Technical Report, UCB/EECS-2009-28, University of California, Berkeley (February 2009)

2. Clark, R.: A Break in the Clouds: Towards a Cloud Definition. ACM SIGCOMM Computer Communication Review 39(1), 50-55 (2009)

3. Shroff, G.: Dev2.0: Model driven development in the Cloud. In: ACM SIGSOFT 2008, Altanta, Gerogiza, USA (2008)

4. Lawton, G.: Developing Software Online with PaaS technology. IEEE Journals Computer 41(6), 13-15 (2008)

5. Mitchell, D.: Defining Platform-As-A-Service, or PaaS, http: / /blogs . bungeeconnect.com/2008/02/18/

6. defining-platform-as-aservice-or-paas/

7. Force.com, http://www. salesforce.com/platform/

8. Compeau, D.R., Higgins, C.A.: Computer self-efficacy: Development of a measure and initial test. MIS Quarterly, pp. 189-211 (June)

9. Salesforce.com. An Introduction to Force.com Apex Code., http: //wiki.developerforce.com/index.php/

An_Introduction_to_Apex;

Salesforce.com. Introduction to Visualforce, http: / /wiki.developerforce.com/index.php/

An_Introduction_to_Visualforce

10. DeveloperForce, http: / / developer. force.com/

11. Weissman, C.D., Bobrowski, S.: The design of the force.com multitenant internet application development platform. In: Proceedings of the 35th SIGMOD international conference on Management of data, SIGMOD 2009 (2009)

12. FormAssembly, http: / / www . formas sembly.com/

13. DabbleDB, http: / / www. dabbledb.com/

14. ZoHo Creator, http: //creator. zoho.com 
15. Wufoo, http://wufoo.com/

16. WaveMaker, http://www . wavemaker.com

17. Chou, D.C., Chou, A.Y.: Analysis of a new information systems outsourcing practice: software-as-a-service business model. International Journal of Information Systems and Change Management 2(4) (2007)

18. JotForm, http: //www. jotform. com

19. QuickBase, http://quickbase.intuit.com/

20. Bungee Connect, http: / / www. bungeeconnect.com/index.html

21. Amazon Web Services, http: / / aws . amazon.com/

22. Google. What Is Google App Engine (2009), http: / / code.google.com/appengine/docs / whatisgoogleappengine.html

23. Salesforce.com. Types of PaaS solutions, http: / /www.salesforce.com/paas/paas-solutions/

24. Facebook, http://www. facebook.com

25. Sledziewski, K., Bordbar, B., Anane, R.: A DSL-Based Approach to Software Development and Deployment on Cloud. In: 24th IEEE International Conferences on Advanced Information Networking and Applications (AINA 2010), Perth, Australia (2010)

26. Boniface, M., Nasser, B., Papay, J., et al.: Platform-as-a-Service Architecture for RealTime Quality of Service Management in Clouds. In: 2010 Fifth International Conference on Internet and Web Applications and Services, Barcelona, Spain (2010)

27. Iceberg, http://www.geticeberg.com/ 